J. Indones. Math. Soc.

Vol. 24, No. 1 (2018), pp. 71-78.

\title{
2-FARTHEST ORTHOGONALITY IN GENERALIZED 2-NORMED SPACES
}

\author{
S. M. Mousav Shams Abad ${ }^{1}$, H. Mazaheri ${ }^{2}$, And M. A. Dehghan ${ }^{3}$ \\ ${ }^{1}$ Faculty of Mathematics, Valiasr Rafsanjan University \\ Rafsanjan, Iran \\ ${ }^{2}$ Faculty of Mathematics, Yazd University \\ Yazd, Iran \\ ${ }^{3}$ Faculty of Mathematics, Valiasr Rafsanjan University \\ Rafsanjan, Iran \\ p92356002@post.vru.ac.ir, hmazaheri@yazd.ac.ir, dehghan@vru.ac.ir
}

\begin{abstract}
In this paper, we consider the concepts 2-farthest orthogonality in generalized 2-normed spaces. We obtain a necessary and sufficient conditions for 2orthogonality of two elements in generalized 2-normed spaces. Also we consider $\epsilon-2-$ farthest orthogonality in generalized 2-normed spaces.

Key words and Phrases: Generalized 2-normed spaces, 2-Bounded sets, 2-Farthest orthogonality, $\epsilon$-2-Farthest orthogonality.
\end{abstract}

\begin{abstract}
Abstrak. Dalam paper ini, kita meninjau konsep Ortogonalitas Terjauh-2 di Ruang bernorma-2 diperumum. Kita mendapatkan syarat perlu dan syarat cukup untuk Ortogonalitas-2 dari dua unsur di ruang bernorma-2 diperumum. Kita juga meninjau Ortogonalitas Terjauh-2- $\epsilon$ di ruang bernorma-2 diperumum.

Kata kunci: Ruang bernorma-2 diperumum, Himpunan terbatas-2, Ortogonalitas terjauh-2, Ortogonalitas terjauh-2- $\epsilon$.
\end{abstract}

\section{INTRODUCTION}

Approximation theory, which mainly consists of theory of nearest points (best approximation) and theory of farthest points (worst approximation), is an old and rich branch of analysis. The theory is as old as Mathematics itself. The ancient Greeks approximated the area of a closed curve by the area of a polygon. Starting in 1853, Russian mathematician P.L. Chebyshev made significant contributions in the theory of best approximation. The Weierstrass approximation theorem of 1885

2000 Mathematics Subject Classification: 46A32 , 46M05, 41A17.

Received: 04-06-2017, revised: 29-07-2017, accepted: 04-09-2017. 
by K. Weierstrass is well known. The study was followed in the first half of the 20th Century by L.N.H. Bunt (1934), T.S. Motzkin (1935) and B. Jessen (1940). B. Jessen was the first to make significant contributions in the theory of farthest points in 1940. This theory is very less developed as compared to the theory of best approximation (see [14]).

Let $X$ be a real vector space with $\operatorname{dim}(X) \geq 2$. A real-valued function $\|.,\|:. X \times X \rightarrow \mathbb{R}$ is called a 2-norm on $X$ if the following conditions hold:

(1) $\|x, y\|=0$ if and only if $x$ and $y$ are linearly dependent.

(2) $\|x, y\|=\|y, x\|$ for all $x, y \in X$.

(3) $\|\alpha x, y\|=|\alpha|\|x, y\|$ for all $\alpha \in \mathbb{R}$ and $x, y \in X$.

(4) $\|x, y+z\| \leq\|x ; y\|+\|x ; z\|$ for all $x, y, z \in X$. The pair $(X ;\|.,\|$.$) is then$ called a 2-normed space.

The 2-norm concept was initially introduced by Gähler in 1960's [4]. Since then, many researchers have developed and obtained various results, see for instance $[5,6,7,11]$. Geometrically, a 2-norm function generalizes the concept of area function of parallelogram due to the fact that, in the standard case, it represents the area of the usual parallelogram spanned by the two associated vectors. Observe that in a 2-normed space we have $\|x, y\|=\|x+\alpha y, y\|$ for any $\alpha \in \mathbb{R}$.

Definition 1.1. [12] Let $X$ and $Y$ be real linear spaces. Denote by $D$ a non-empty subset of $X \times Y$ such that for every $x \in X, y \in Y$ the sets $D_{x}=\{y \in Y:(x, y) \in$ $D\}$ and $D_{y}=\{x \in X:(x, y) \in D\}$ are linear subspaces of the spaces $Y$ and $X$, respectively. A function $\|.,\|:. D \rightarrow[0, \infty)$ will be called a generalized 2-norm on $D$ if it satisfies the following conditions:

(1) $\|x, \alpha y\|=|\alpha| .\|x, y\|=\|\alpha x, y\|$ for any real number $\alpha$ and all $(x, y) \in D$;

(2) $\|x, y+z\| \leq\|x, y\|+\|x, z\|$ for $x \in X, y, z \in Y$ with $(x, y),(x, z) \in D$;

(3) $\|x+y, z\| \leq\|x, z\|+\|y, z\|$ for $x, y \in X, z \in Y$ with $(x, z),(y, z) \in D$. The set $D$ is called a 2-normed set.

In particular, if $D=X \times Y$, the function $\|.,$.$\| is said to be a generalized 2-$ norm on $X \times Y$ and the pair $(X \times Y,\|.,\|$.$) is called a generalized 2-normed space. If$ $X=Y$, then the generalized 2-normed space $(X \times X,\|.,\|$.$) is denoted by (X,\|.,\|$.$) .$ In the case that $X=Y$ and $D=D^{-1}$, where $D^{-1}=\{(y, x):(x, y) \in D\}$, and $\|x, y\|=\|y, x\|$ for all $(x, y) \in D$, we call $\|.,$.$\| a generalized symmetric 2$-norm and $D$ a symmetric 2-normed set.

Recall that in Gähler definition of a 2-norm $\|x, y\|=0$ if and only if $x$ and $y$ are linearly dependent, and this is a crucial difference between Gählerfs approach and Lewandowska's one.

Example 1.2. [12] Let $X$ be a real linear space having two seminorms $\|\cdot\|_{1}$ and $\|.\|_{2}$. Then $(X,\|.,\|$.$) is a generalized 2-normed space with the 2-norm defined by$

$$
\|x, y\|=\|x\|_{1} \cdot\|y\|_{2} ; x, y \in X .
$$

Every 2-normed space is a locally convex topological vector space. In fact for a fixed $b \in X, p_{b}=\|x, b\| ; x \in X$ is a semi-norm on $X$ and the family 
$P=\left\{p_{b}: b \in X\right\}$ of semi-norms generates a locally convex topology. We know in a topological vector space $(X, \tau)$ over a field $F, W$ is called bounded if for every neighborhood $N$ of the zero vector there exists a scalar $\alpha$ such that

$$
W \subseteq \alpha N
$$

with $\alpha N:=\{\alpha x \mid x \in N\}$.

In 2-normed spaces, the set $W$ is 2-bounded set if $\sigma(M)<\infty$, where

$$
\sigma(M)=\sup \{\|x-z, y-z\|: x, y, z \in M\} .
$$

Let $(X,\|.,\|$.$) be a 2-normed space and let W_{1}$ and $W_{2}$ be two subspaces of $X$. A map $f: W_{1} \times W_{2} \rightarrow \mathbb{R}$ is called a bilinear 2 -functional on $W_{1} \times W_{2}$ whenever for all $x_{1}, x_{2} \in W_{1}, y_{1}, y_{2} \in W_{2}$ and all $\lambda_{1}, \lambda_{2} \in \mathbb{R}$;

(i) $f\left(x_{1}+x_{2}, y_{1}+y_{2}\right)=f\left(x_{1}, y_{1}\right)+f\left(x_{1}, y_{2}\right)+f\left(x_{2}, y_{1}\right)+f\left(x_{2}, y_{2}\right)$,

(ii) $f\left(\lambda_{1} x_{1}, \lambda_{2} y_{1}\right)=\lambda_{1} \lambda_{2} f\left(x_{1}, y_{1}\right)$.

A bilinear 2-functional $f: W_{1} \times W_{2} \rightarrow \mathbb{R}$ is called bounded if there exists a non-negative real number $M$ (called a Lipschitz constant for $f$ ) such that $|f(x, y)| \leq$ $M\|x, y\|$ for all $x \in W_{1}$ and all $y \in W_{2}$. Also, the norm of a bilinear 2-functional $\mathrm{f}$ is defined by

$$
\|f\|=\inf \{M \geq 0: M \text { is a Lipschitz constant } f\} .
$$

It is known that

$$
\begin{aligned}
\|f\| & =\sup \left\{|f(x, y)|:(x, y) \in W_{1} \times W_{2},\|x, y\| \leq 1\right\} \\
& =\sup \left\{|f(x, y)|:(x, y) \in W_{1} \times W_{2},\|x, y\|=1\right\} \\
& =\sup \left\{\frac{|f(x, y)|}{\|x, y\|}:(x, y) \in W_{1} \times W_{2},\|x, y\|>0\right\} .
\end{aligned}
$$

For a generalized 2-normed space $(X,\|.,\|$.$) and 0 \neq b \in X$, we denote by $X_{b}^{*}$, the Banach space of all bounded bilinear 2-functionals on $X \times<b>$, where $\langle b\rangle$ be the subspace of $X$ generated by $b$.

\section{2-Farthest Orthogonality in Generalized 2-Normed Spaces}

In this section we consider farthest orthogonality in generalized 2-normed spaces.

Definition 2.1. Let $(X,\|.,\|$.$) be a generalized 2-normed space. For x, y \in X$, we say that $x$ is 2-farthest orthogonality to $y$ and denote by $x \perp_{2 F} y$, if for all $z \in X$,

$$
\|x, z\| \geq\|x-y, z\| \text { and }\|x-y, z\| \neq 0 .
$$

If $W$ a subset of $X$ and $x \in X$, we say that $x \perp_{2 F} W$ if and only if $x \perp_{2 F} y$. for every $y \in W$. 
Let $(X,\|.,\|$.$) be a generalized 2-normed space and W$ be bounded subset of $X$. A point $y_{0} \in W$ is said to be a 2 -farthest point for $x \in X$, if

$$
\left\|x-y_{0}, z\right\| \geq\|x-y, z\| \text { for all } z \in X \text { and for all } y \in W .
$$

If $f_{W}(x, z)=\sup _{y \in W}\|x-y, z\|$, then $y_{0}$ is 2-farthest point of $x$, if $\left\|x-y_{0}, z\right\|=$ $f_{W}(x, z)$ for all $z \in X$. For all $y \in W$, put

$$
F_{W}^{2}(x)=\left\{y_{0} \in W:\left\|x-y_{0}, z\right\|=f_{W}(x, z) \text { for all } z \in X\right\} .
$$

For $x \in X$, if $F_{W}^{2}(x)$ is nonempty, we say that $W$ is 2-remotal. For $x \in X$, if $F_{W}^{2}(x)$ is singleton, we say that $W$ is 2-uniquely remotal. For $x \in X$, if $F_{W}^{2}(x)=\emptyset$, we say that $W$ is 2-anti-remotal.

A sequence $\left\{x_{n}\right\}_{n \geq 1}$ in a generalized 2-normed space $(X,\|.,\|$.$) is called a$ convergent sequence if there exists an $x \in X$ such that $\lim _{n \rightarrow \infty}\left\|x_{n}, z\right\|=\|x, z\|$ for all $z \in X$. If $\left\{x_{n}\right\}_{n \geq 1}$ converges to $x$, we write $x_{n} \rightarrow x$ as $n \rightarrow \infty$.

Theorem 2.2. Let $(X,\|.,\|$.$) be a generalized 2$-normed space and $x, y \in X$. If for some $k \in \mathbb{R} \backslash\{0\}, y=k x$ and $x \perp_{2 F} y$, then $0 \leq k \leq 2$.

Proof. Suppose $y=k x$, Therefore $\|x, z\| \geq\|(1-k) x, z\|$ for all $z \in X$. It follows that $1 \geq|1-k|$. Hence $0 \leq k \leq 2$.

Theorem 2.3. Let $(X,\|.,\|$.$) be a generalized 2-norm and x, t \in X$. For all $z \in X$, if $\|x, z\| \geq\|x-t, z\|$, then for every $k \geq 1, k\|x, z\| \geq\|k x-t, z\|$, that is, if $x \perp_{2 F} t$, then for every $k \geq 1, k x \perp_{2 F} y$.

Proof. Suppose $z \in X$, define $F_{z}:[0, \infty) \rightarrow \mathbb{R}$ by $F_{z}(k)=\|k x-t, z\|-\|k x, z\|$. Then $F_{z}$ is convex function such that $F_{z}(0)=\|t, z\| \geq F_{z}(k)$ for every $k \in \mathbb{R}$, $F_{z}(1)=\|x-t, z\|-\|x, z\| \leq 0$. Thus we have $F_{z}(k) \leq 0$ for every $k \geq 1$.

Example 2.4. Suppose $X=\mathbb{R}^{2}$ with the 2 -norm $\|$. . . $\|$ defined by $\left\|\left(a_{1}, a_{2}\right),\left(b_{1}, b_{2}\right)\right\|=$ $\left|a_{1} b_{2}-a_{1} b_{1}\right|$ is a 2-norm. Then $(3,3) \perp_{2 F}(2,2)$.

Proposition 2.5. Let $(X,\|.,\|$.$) be a 2-normed space. Then:$

(i) If $x \in X$, then $x \perp_{2 F} 0$.

(ii) If $x \perp_{2 F} y$ and $\|x, z\|=\|y, z\|$, for all $z \in X$, then $y \perp_{2 F} x$.

(iii) If $0 \perp_{2 F} x$ for $x \in X$, then $x=0$.

(vi) For $\alpha \in \mathbb{R}, x \perp_{2 F} y$ if and only if $\alpha x \perp_{2 F} \alpha y$.

(v) If $x_{n} \rightarrow x, y_{n} \rightarrow y$ and $x_{n} \perp_{2 F} y_{n}$, then $x \perp_{2 F} y$.

Proof. It is trivial. 
Theorem 2.6. Let $(X,\|.\|$,$) be a generalized 2-norm and x, y \in X$. Then the following statements are equivalent:

(1) $x \perp_{2 F} y$,

(2)for every $z \in X$, there exists a 2-bilinear functional $T_{z} \in X_{z}^{*}$ such that $\left|T_{z}(x, z)\right| \geq\|x-y, z\|$ and $\left\|T_{z}\right\|=1$.

Proof. $(2) \Rightarrow(1)$. For every $z \in X$, suppose that there is a 2-bilinear functional $T_{z} \in X_{z}^{*}$ such that $\left|T_{z}(x, z)\right| \geq\|x-y, z\|$ and $\left\|T_{z}\right\|=1$. Then

$$
\begin{aligned}
\|x, z\| & =\|x, z\|\left\|T_{z}\right\| \\
& \geq\left|T_{z}(x, z)\right| \\
& \geq\|x-y, z\| .
\end{aligned}
$$

$(1) \Rightarrow(2)$. Conversely suppose that $x \perp_{2 F} y$ and $z \in X$. For all $z \in X$, we have $\|x-y, z\| \neq 0$, it follows that $\|x, z\| \neq 0$. From Hahan Banach Theorem in the context of 2-normed spaces (see Theorem $2.2[12]$ ), there exists a bilinear $T_{z} \in X_{z}^{*}$, such that $\left\|T_{z}\right\|=1$ and $\left|T_{z}(x, z)\right|=\|x, z\| \geq\|x-y, z\|$.

Theorem 2.7. Let $(X,\|.,\|$.$) be a generalized 2-norm, W$ a bounded subset of $X$ and $x \in X$. If for all $z \in X$, there exists a 2-bilinear functional $T_{z} \in X_{z}^{*}$ such that $\left|T_{z}(x, z)\right|=f_{W}(x, z)$ and $\left\|T_{z}\right\|=1$, then $x \perp_{2 F} W$,

Proof. For $z \in X$ and $y \in W, f_{W}(x, z) \geq\|x-y, z\|$. From Theorem 2.2, $x \perp_{2 F} y$. Therefore $x \perp_{2 F} W$.

Theorem 2.8. Let $(X,\|.,\|$.$) be a generalized 2-norm and W$ a bounded subset of $X$. If $g_{1} \in F_{W}^{2}(x), g_{2} \in F_{W}^{2}(y)$ and $z \in X$,

(i) $2\|x-y, z\| \leq\left\|x-g_{1}, z\right\|+\left\|y-g_{2}, z\right\|$,

(ii) If $g_{1}-W=W$, then $x-g_{1} \perp_{2 F} W$.

Proof. (i) From definition of 2-farthest points, we have for every $z \in X$

$$
\|x-y, z\| \leq\left\|x-g_{1}, z\right\|,\|x-y, z\| \leq\left\|y-g_{1}, z\right\| .
$$

Therefore $2\|x-y, z\| \leq\left\|x-g_{1}, z\right\|+\left\|y-g_{2}, z\right\|$.

(ii) For $y \in W$,

$$
\begin{aligned}
\left\|x-g_{1}, z\right\| & \geq\|x-y, z\| \\
& =\left\|x-g_{1}+g_{1}-y, z\right\| .
\end{aligned}
$$

Suppose $g_{1}-y=u$, then $u \in W$. Therefore $x-g_{1} \perp_{2 F} W$.

Theorem 2.9. Let $(X,\|.,\|$.$) be a generalized 2-norm, W$ a bounded subset of $X$ and $g_{0} \in W$. Then the set $\left(F^{2}{ }_{W}\right)^{-1}\left(g_{0}\right)=\left\{x \in X: g_{0} \in F_{W}^{2}(x)\right\}$ is closed.

Proof. Suppose $\left\{x_{n}\right\}_{n \geq 1}$ any sequence in $\left(F^{2} W\right)^{-1}\left(g_{0}\right)$ and $x \in X$ such that $\lim _{n \rightarrow \infty} x_{n}=x$. Then for every $z \in X$ and $g \in W$ and for $n \geq 1$, we have

$$
\left\|x_{n}-g_{0}, z\right\| \geq\left\|x_{n}-g, z\right\| .
$$


By limiting to $\infty$, we have for every $z \in X$ and for every $g \in W\left\|x-g_{0}, z\right\| \geq$ $\|x-g, z\|$. That is $x \in\left(F^{2} W\right)^{-1}\left(g_{0}\right)$, and $\left(F^{2} W\right)^{-1}\left(g_{0}\right)$ is closed.

Corollary 2.10. Let $(X,\|.,\|$.$) be a generalized 2-norm and y_{0} \in X$. Put $E_{y_{0}}=$ $\left\{x \in X: x \perp_{2 F} y_{0}\right\}$, then $E_{y_{0}}$ is closed in $X$.

Proof. Suppose $\left\{x_{n}\right\}_{n \geq 1}$ any sequence in $E_{y_{0}}$ and $x \in X$ such that $\lim _{n \rightarrow \infty} x_{n}=$ $x$. Therefore for every $z \in X, \lim _{n \rightarrow \infty}\left\|x_{n}, z\right\|=\|x, z\|$. Since $x_{n} \perp_{2 F} y_{0}$, for every $n \geq 1,\left\|x_{n}, z \mid \geq\right\| x_{n}-y_{0}, z \|$. Therefore $\|x, z\| \geq\left\|x-y_{0}, z\right\|$. It follows that $x \perp_{2 F} y_{0}$.

\section{3. $\epsilon$-2-FARThest Orthogonality}

In this section we consider $\epsilon$-2-farthest points in generalized 2-norm spaces.

Definition 3.1. Let $(X,\|.\|$,$) be a generalized 2-norm space and x, y \in X$ and $\epsilon>0$. We say that $x \perp_{2 F}^{\epsilon} y$, if for all $z \in X$,

$$
\|x, z\| \geq\|x-y, z\|-\epsilon, \text { and }\|x-y, z\| \neq \epsilon .
$$

Also, if $W$ is a subset of $X$ and $x \in X$, then $x \perp_{2 F}^{\epsilon} W$, if $x \perp_{2 F}^{\epsilon} y$ for every $y \in W$. Put

$$
F_{W, \epsilon}^{2}(x)=\left\{y_{0} \in W:\left\|x-y_{0}, z\right\| \geq f_{W}(x, z)-\epsilon \text { for all } z \in X\right\} .
$$

Theorem 3.2. Let $(X,\|.,\|$.$) be a generalized 2-norm, x, y \in X$ and $\epsilon>0$. Then the following statements are equivalent:

(1) $x \perp_{2 F}^{\epsilon} y$,

(2) for all $z \in X$, there exists a 2-bilinear functional $T_{z} \in X_{z}^{*}$ such that $\left|T_{z}(x, z)\right| \geq\|x-y, z\|-\epsilon$ and $\left\|T_{z}\right\|=1$.

Proof. $(2) \Rightarrow(1)$. For every $z \in X$, suppose that there is a 2-bilinear functional $T_{z} \in X_{z}^{*}$ such that $\left|T_{z}(x, z)\right| \geq\|x-y, z\|-\epsilon$ and $\left\|T_{z}\right\|=1$. Then

$$
\begin{aligned}
\|x, z\| & =\|x, z\|\left\|T_{z}\right\| \\
& \geq\left|T_{z}(x, z)\right| \\
& \geq\|x-y, z\|-\epsilon .
\end{aligned}
$$

$(1) \Rightarrow(2)$. Conversely, suppose $x \perp_{2 F}^{\epsilon} y$ and $z \in X$. For all $z \in X$, we have $\|x-y, z\| \neq \epsilon$, it follows that $\|x, z\| \neq 0$. From Hahan Banach Theorem in the context of 2-normed spaces (see Theorem $2.2[12]$ ), there exists a bilinear $T_{z} \in X_{z}^{*}$, such that $\left\|T_{z}\right\|=1$ and $\left|T_{z}(x, z)\right|=\|x, z\| \geq\|x-y, z\|-\epsilon$.

Theorem 3.3. Let $(X,\|.,\|$.$) be a generalized 2-norm, W$ a subset of $X, x \in X$ and $\epsilon>0$. If for all $z \in X$, there exists a 2-bilinear functional $T_{z} \in X_{z}^{*}$ such that $\left|T_{z}(x, z)\right|=f_{W}(x, z)-\epsilon$ and $\left\|T_{z}\right\|=1$, then $x \perp_{2 F}^{\epsilon} W$,

Proof. For $z \in X$ and $y \in W, f_{W}(x, z)-\epsilon \geq\|x-y, z\|-\epsilon$. From above Theorem, $x \perp_{2 F}^{\epsilon} y$. Therefore $x \perp_{2 F}^{\epsilon} W$. 
Theorem 3.4. Let $(X,\|.,\|$.$) be a generalized 2-norm and W$ a subset of $X$. If $g_{1} \in F_{W, \epsilon}^{2}(x), g_{2} \in F_{W, \epsilon}^{2}(y)$ and $z \in X$, then
(i) $2(\|x-y, z\|-\epsilon) \leq\left\|x-g_{1}, z\right\|+\left\|y-g_{2}, z\right\|$,
(ii) If $g_{1}-W=W$, then $x-g_{1} \perp_{2 F}^{\epsilon} W$.
(iii) For $\alpha \geq 0, x \perp_{2 F}^{\epsilon} y$ if and only if $\alpha x \perp_{2 F}^{\alpha \epsilon} \alpha y$

Proof. It is trivial.

Definition 3.5. Let $(X,\|.\|$,$) be a generalized 2-norm and G$ and $A$ be a nonempty subset of $X, z \in X$. A point $g_{0} \in G$ is called a simultaneous $z$-farthest point to $A$ from $G$ if

$$
\rho_{z}(A, G)=\sup _{g \in G} \sup _{a \in A}\|g-a, z\|=\sup _{a \in A}\left\|g_{0}-a, z\right\| .
$$

Put

$$
F_{G}^{z}(A)=\left\{g \in G: \sup _{a \in A}\|g-a, z\|=\rho_{z}(A, G)\right\} .
$$

Theorem 3.6. Let $(X,\|.,\|$.$) be a generalized 2-norm and G$ and $A$ be a non-empty subset of $X, z \in X$. If $g \in F_{G}^{z}(A) \cap L(y, W)$ for some $w, y \in G$. Then $y, w \in F_{G}^{z}(A)$, where $L(y, W)=\{t y+(1-t) W: 0<t<1\}$.

Proof. Suppose $g \in F_{G}^{z}(A) \cap L(y, w)$ for some $w, y \in G$. Then $\sup _{a \in A}\|g-a, b\|=$ $\rho_{z}(A, G)$ and $g=t y+(1-t) w$ for some $t \in[0,1]$. Further

$$
\begin{aligned}
\sup _{a \in A}\|g-a, b\| & =\|t y+(1-t) w-a, z\| \\
& =\sup _{a \in A}\|t(y-a)+(1-a)(w-a), z\| \\
& \leq t \sup _{a \in A}\|y-a, z\|+(1-t) \sup _{a \in A}\|w-a, z\| \\
& \leq t \rho_{z}(A, G)+(1-t) \rho_{z}(A, G) \\
& =\rho_{z}(A, G) .
\end{aligned}
$$

We assume that $\sup _{a \in A}\|y-a, z\|<\rho_{z}(A, G)$ or $\sup _{a \in A}\|w-a, z\|<\rho_{z}(A, G)$. Then $\rho_{z}(A, G)<\rho_{z}(A, G)$, which is contradicition. Therefore

$$
\sup _{a \in A}\|y-a, z\|=\rho_{z}(A, G)=\sup _{a \in A}\|w-a, z\|=\sup _{a \in A}\|g-a, z\| .
$$

Therefore $y, w \in F_{G}^{z}(A)$.

\section{REFERENCES}

[1] Cho, Y.J. and Kim, S.S., "Gateaux Derivatives and 2-Inner Product" Glas. Mat. Ser. III, 27(1983), 197-203.

[2] Diminnie, C.R. and White, A., "A Characterization of 2-Inner Product Spaces", Math. Japon., 114(1983), 275-277.

[3] Dragomir, S.S., Cho, Y.J. and Kim, S.S., "Superadditivity and Monotonicity of 2-Norms Generated by Inner Products and Related Results", Soochow J. Math., 24(1998), 13-32. 
[4] Gähler, S., "Lineare 2-Normierte Raume", Math. Nachr., 28(1964), 1-43.

5] Gozali, S.M. and Gunawan, H. On b-orthogonality in 2-normed spaces. J. Indones. Math. Soc., 16(2010), 127-132.

[6] Gunawan, H., "On n-Inner Products, n-Norms, and the Cauchy-Schwarz Inequality", Sci. Math. Jpn., 55(2002), 53-60.

[7] Gunawan, H., "Inner Product on n-Inner Product Spaces", Soochow J. Math., 28(2002), 389-398.

[8] Gunawan, H. and Mashadi, "On n-Normed Spaces", Int. J. Math. Math. Sci., 27(2001), 631-639.

[9] Gunawan, H., Mashadi, Gemawati, S., Nursupiamin and Sihwaningrum, I., "Orthogonality in 2-Normed Spaces Revisited", Univ. Beograd Publ. Elektrotehn. Fak. Ser. Mat., 17(2006), $76-83$.

[10] Kamali, H.R. and Mazaheri, H., "b-General Orthogonality in 2-Normed Spaces", Int. Appl. Math. Sci., 2(2008), 775-780.

[11] Khan, A. and Siddiqui, A., "B-Orthogonality in 2-Normed Space", Bull. Calcutta Math. Soc., 7:4(1982), 216-222.

[12] Konca, S., Idris, M. and Gunawan, H. "A new 2-inner product on the space of p-summable sequences", Journal of the Egyptian Mathematical Society, 24:2(2016), 244-249.

[13] Konca, S., Idris, M., Gunawan, H. and Basarir, M., "p-Summable sequence spaces with 2-inner products", Contemporary Analysis and Applied Mathematics, 3(2015), 213-226.

[14] Lewandowska, Zoa, Moslehian, Mohammad Sal; Saadatpour M., Assieh. "Hahn-Banach theorem in generalized 2-normed spaces." Commun. Math. Anal., 1(2006), 109-113.

[15] Mazaheri, H. and Kazemi, R., "Some Results on 2-Inner Product Spaces", Novi Sad J. Math., 37(2007), 35-40

[16] Mazaheri, H., Narang, T.D., and Khademzadeh, H.R., Nearest and Farthest points in normed spaces, In Press Yazd University, 2015 